\title{
Proceedings of the 146th Semon Club, 5 November 2013, ENT Department, Guy's and St Thomas' NHS Foundation Trust, London, UK
}

\author{
Chairman: Miss Elfy B Chevretton, Guy's and St Thomas' NHS Foundation Trust \\ Secretary: Mr Sherif Haikel, Royal National Throat, Nose and Ear Hospital, University College London Hospitals NHS Trust \\ Invited panel for pathology: Dr Ann Sandison, Charing Cross Hospital, Imperial College Healthcare NHS Trust \\ Invited panel for radiology: Dr Steve Connor, and Dr Ata Siddiqui, Guy's and St Thomas' NHS Foundation Trust \\ The Professor Leslie Michaels prize for the best presentation of the meeting was awarded to Carly Grandidge for "“Pituitary palsy" - progressive \\ neck pain and arm weakness following trans-sphenoidal hypophysectomy'. \\ The chairman and secretary have edited the proceedings of the meeting to reflect the discussion of each case by the expert panel and audience \\ during the Semon Club meeting.
}

\section{Otology section}

Chairman: Mr Dan Jiang

A unique case of cochlear implantation in Hallermann-Streiff syndrome

K L Whitcroft, C Pepper, D Selvadurai

From St George's Hospital, London

\section{Introduction}

Hallermann-Streiff syndrome is a rare genetic condition involving ocular, mandibular and facial anomalies. We present the only documented case of profound hearing loss and cochlear implantation in a patient with Hallermann-Streiff syndrome.

\section{Case report}

A 47-year-old woman with Hallermann-Streiff syndrome and learning difficulties presented with progressive hearing loss. Otoscopy was largely unremarkable, and audiological assessment confirmed bilateral profound sensorineural hearing loss, with no benefit from hearing aids. Magnetic resonance imaging (MRI) and computed tomography (CT) scans were performed. Her case was discussed in the cochlear implant multidisciplinary team meeting where it was decided to proceed with cochlear implantation.

\section{Radiology}

The MRI scan confirmed the presence of normal vestibulocochlear nerves, and revealed a high T2-weighted signal in both middle ears. The CT scan showed widely abnormal skull base anatomy, with poorly developed and abnormal mastoids. Opacity was present in both middle ears, suggesting the possibility of cholesteatoma.

In light of the abnormal temporal bone anatomy and the possibility of cholesteatoma, the patient initially underwent right radical mastoidectomy. This confirmed adequate access to the round window and the presence of granulation tissue.

\section{Histopathology}

Granulation tissue from the right mastoid showed cholesterol clefts and haemosiderin pigment deposition, but no squamous epithelium or keratin. Cholesteatoma was thought unlikely.

Subsequently, a staged cochlear implantation and blind sac closure procedure were undertaken. The patient's recovery from these procedures was uneventful, and the cochlear implant was functional.

\section{Discussion and lessons learnt}

Dr Connor described the anatomical abnormalities of the temporal bones that made implantation difficult in this case. It was concluded that implantation in such cases is achievable with careful pre-operative planning and a multidisciplinary approach. Mr Jiang mentioned that he would have completed the blind sac closure at the time of the first procedure (performing a radical mastoidectomy).

An unusual cause of chronic suppurative otitis media

R Advani, R Siripurapu, S Agrawal

From the Pennine Acute Hospitals NHS Trust, Manchester

\section{Introduction}

Pyogenic granuloma was first described in 1897 and has rarely been reported in the middle ear.

\section{Case report}

A 48-year-old man presented with a 20 -year history of otorrhoea, hearing loss and a polyp obstructing his medial external auditory canal.

\section{Radiological findings}

Radiological imaging showed an intact scutum, complete erosion of the incus, bony erosion of the antrum and fluid in the mastoid. Dr Connor suggested the findings were consistent with a benign pathology.

\section{Histological findings}

Dr Sandison noted the polypoidal tissue with surface ulceration. Vascular oedematous inflammatory tissue was observed. This is consistent with granulation tissue or a pyogenic granuloma. Dr Sandison mentioned that 'lobular capillary haemangioma' would be another name for such a proliferation of blood vessels and inflammatory tissue. 


\section{Management}

Combined approach tympanoplasty was performed for presumed mucosal disease. The antrum was free of disease. Firm vascular tissue was found in the mesotympanum, extending into the external auditory canal. Following dissection, conchal cartilage and bone pate were used to graft the tympanic membrane and obliterate the mastoid cavity respectively.

\section{Discussion}

Mr Jiang enquired as to whether the patient had undergone previous ear surgery and was informed that he had not. He commented that the granulation tissue in the middle ear may have resulted from multiple attempts at microsuction and packing over a prolonged period of time.

\section{Conclusion}

Although common in the head and neck, pyogenic granuloma rarely occurs in the middle ear. Radiological appearances are inconsistent and diagnosis is based on histological analysis.

\section{A middle-ear tumour demonstrating a range of distinct histological patterns}

H Blanchford, L Lindsey, P Sloan

From the Freeman Hospital, Newcastle upon Tyne

\section{Introduction}

Middle-ear adenomas are rare, benign tumours that demonstrate variable differentiation along neuroendocrine and epithelial pathways. Glomus tympanicum is the term ascribed to more commonly occurring paragangliomas that originate from the tympanic cavity.

\section{Case report}

A 46-year-old man presented with a 12-month history of a painful, discharging ear. An aural polyp was identified, which obstructed the external auditory canal. Pure tone audiometry revealed unilateral mixed hearing loss. The tumour extended from the middle ear into the external auditory canal via an attic perforation. Initial histology suggested a benign vascular lesion, most likely representing a glomus tympanicum. The tumour was found encasing the ossicular chain. Radical mastoidectomy was performed.

\section{Radiological findings}

Computed tomography demonstrated an intact ossicular chain surrounded by abnormal soft tissue, with complete opacification of the middle ear and mastoid air cells. A small area of calcification was observed, separate from the ossicles. Dr Connor mentioned that an enhanced magnetic resonance imaging scan would allow identification of soft tissue and fluid.

\section{Histological findings}

The glomus tympanicum diagnosis was questioned when glandular features were found in further specimens. Immunohistochemically, tumour cells were positive for both cytokeratin markers and synaptophysin (a neuroendocrine marker). Tumour cells demonstrated unusual plasmacytoid features and were arranged in a range of architectural patterns.

\section{Management}

Management of middle-ear adenomas requires surgical resection to prevent bony erosion and cranial nerve involvement. At 18 months, our patient had no tumour recurrence.

\section{Discussion}

Dr Sandison agreed with the diagnosis of middle-ear adenoma, noting the arrangement of cells in coils and the absence of sustentacular cells. She commented that recurrence is rare if the tumour is completely excised.

Dr Sandison also felt that both tumours were likely to originate from neuroendocrine stem cells, and with developments in immunostaining, our understanding of the origins of these tumours may improve. Professor Michaels had previously shown that adenomas were not carcinoid tumours

\section{Conclusion}

Middle-ear adenoma remains a diagnostic challenge, demonstrated here by the preliminary diagnosis of glomus tympanicum. Diagnosis is complicated by the fact that adenomas can demonstrate many distinct histological patterns in a single tumour.

Otorrhoea, 'dead ear' and facial palsy following auditory implantation

E Warner, S Connor, D Jiang

From the Guy's and St Thomas' NHS Foundation Trust, London

\section{Introduction}

We present two cases whereby middle- and inner-ear surgery provided an access route for infection of the labyrinth. Chronic inflammation in this area usually leads to 'labyrinthine ossificans', resulting in ossification and fibrosis. Rarely, non-resolving infection occurs due to ongoing access to the labyrinth, and osteonecrotic changes (labyrinthine sequestration) result. This pathology has never previously been reported following auditory implantation surgery.

\section{Case report}

Case one was a 53-year-old, fit and well patient, who presented with persistent otorrhoea and symptoms of otitis externa following stapedectomy. Case two was an immunosuppressed 75-year-old, who developed otorrhoea and disequilibrium several years after a cochlear implantation.

After treatment with combinations of antibiotics and steroids for some time, the symptoms progressed. Case one developed sudden onset sensorineural hearing loss. Case two developed sudden onset facial palsy. Computed tomography (CT) scans of case two showed the extremely unusual findings of diffuse osteolysis related to the otic capsules (with some minor bony 'sequestration'), whilst magnetic resonance imaging of case one revealed diffuse labyrinthine enhancement. Histology showed features consistent with chronic inflammation.

Case one had his implant removed, and the infection resolved. Surgical intervention was precluded in case two because of co-morbid disease; interestingly, there was spontaneous resolution of the radiological changes, with followup CT showing labyrinthine ossification.

\section{Conclusions}

To our knowledge, there are no previously reported cases of surgical implants acting as a portal for tympanogenic 
labyrinthitis. Previous authors (e.g. Lao, 2012) have advocated aggressive surgical approaches for management of these cases; however, we witnessed resolution in our second case without surgery.

A rare cause of unilateral deafness, otalgia and facial weakness

R Sproat, M Bull, O Whiteside

From Wexham Park Hospital, Slough

Introduction

Keratosis obturans is the accumulation of desquamated keratin in the external auditory canal. We describe an unusual presentation of keratosis obturans.

\section{Case}

A 35 -year-old woman who was 25 -weeks pregnant presented with a 3 -week history of significant right otalgia, deafness and facial weakness. She had House-Brackmann grade II right facial nerve palsy. Otoscopy revealed an expanded right external auditory canal filled with a large mass of keratin. Clear pulsatile otorrhoea was noted. Full clearance was carried out under local anaesthetic. Computed tomography (CT) was performed to rule out a cerebrospinal fluid (CSF) leak.

An unenhanced CT scan of the patient's right temporal bone showed marked widening and erosion of the external canal, with a focus of erosion extending to the mastoid canal of the facial nerve and no dural leak.

Histological examination confirmed keratin flakes.

\section{Management}

The facial nerve palsy resolved after two months with regular microsuction and EarCalm Spray ${ }^{\circledR}$.

\section{Discussion}

The distinction between external auditory canal cholesteatoma and keratosis obturans is controversial. This was demonstrated by the ensuing discussion.

Dr Sandison commented that a histological distinction cannot be made between the two entities. The normal ear canal contains keratinising epithelium, and therefore Dr Sandison prefers to avoid the term cholesteatoma of the ear canal; however, she felt the distinction between the two conditions was clinical and not pathological.

Mr Jiang felt that the two conditions were very different clinically, with keratosis obturans being more aggressive, causing diffuse bony erosion and more symptoms for the patient. External auditory canal cholesteatoma, however, did not cause inflammation of the external auditory canal skin, and was more likely to result in localised bony erosion. Mr Jiang commented that there was evidence of localised erosion over the facial nerve, drawing him towards the diagnosis of external auditory canal cholesteatoma.

Mr Haikel suggested that tests of $\beta-2$ transferrin levels could have been carried out on the clear otorrhoea to exclude a CSF leak, in order to avoid performing a CT scan on a pregnant patient.

The circumferential enlargement of the external auditory canal led the authors to conclude that this was likely to be a case of keratosis obturans.

\section{Conclusion}

This is the third described case of probable keratosis obturans associated with facial nerve palsy.

\section{Rhinology section}

Chairman: Miss Jo Rimmer

\section{An unusual lump of the nasal alar}

A S Takhar, J Stephens, P Andrews

From the Royal National Throat, Nose and Ear Hospital, University College London Hospital NHS Trust

\section{Introduction}

Angioleiomyoma is a rare, benign, vascular, smooth muscle tumour. Nasal vascular leiomyomas are rare, with only 37 reports in the English language literature; most were found in females and were located within the nasal cavity. We report what we believe to be the first ever case of a subcutaneous angioleiomyoma of the nasal alar in a male.

\section{Case report}

A 56-year-old gentleman presented with a painless, subcutaneous, erythematous lump of the right nasal alar, which had been slowly growing for 6 years. This had led to complete unilateral nasal obstruction and poor cosmesis. Surgical excision was undertaken using an external rhinoplasty approach.

\section{Radiological findings}

Magnetic resonance imaging (MRI) demonstrated an ovalshaped lesion with flow voids indicating vascularity. The lesion measured $2.3 \times 1.8 \times 1.4 \mathrm{~cm}$. It was isointense on T1-weighted images and of high-signal intensity on T2weighted images.

\section{Histological findings}

Microscopic evaluation demonstrated a well-circumscribed nodule composed of variably sized vessels and surrounded by concentric bundles of smooth muscle cells. There was no evidence of atypia or malignancy.

Discussion on the day

Dr Connor explained that MRI has little specificity in the diagnosis of such lesions and MRI angiography may have been useful. During the meeting, Miss Rimmer asked if the lesion had any malignant potential. Dr Sandison responded that there was no evidence for this.

\section{Conclusion and take-home message}

Because of the rarity of this lesion, extensive pre-operative planning was required. Blood was cross-matched and the patient consented to undergo conchal cartilage harvest for potential external nasal valve reconstruction. However, the lesion was easily excised with minimal blood loss, and no reconstruction was required. The functional and cosmetic result was excellent.

Bilateral nasal blockage and anosmia: not just another nasal polyp

E Warner, P R Morgan, C Hopkins

From the Guy's and St Thomas' NHS Foundation Trust, London

\section{Case report}

A 34-year-old man with no significant past medical history presented with a 5-month history of progressive bilateral nasal obstruction. He was treated with nasal and oral steroids with no response. Examination revealed bilateral nasal polyps with a benign appearance. 


\section{Imaging}

The computed tomography images were inconsistent with a typical case of polyps - the polypoid lesion arose centrally with destruction of the septum, whilst the sinuses were completely clear.

\section{Histology}

Endoscopic resection was undertaken. Histology revealed the unusual diagnosis of glomangiopericytoma (sinonasal haemangiopericytoma). Dr Sandison noted how parts of the polyp had histological features identical to an inflammatory polyp, which could explain the initial diagnosis following clinical examination. The patient was referred to a tertiary hospital for complete surgical resection following the discovery of residual disease. Further expert histopathological opinion was sought because of the unusual immunochemical and histological features. These features included increased Ki-67 staining, which suggested a more aggressive phenotype of this usually indolent tumour.

\section{Management}

Given the more aggressive nature of the tumour, the patient will be followed up closely, with magnetic resonance imaging scans performed quarterly. In the event of recurrence, resection of the hard palate may be required.

\section{Discussion}

Haemangiopericytoma, which was first described by Stout and Murray in 1942, is a rare vascular tumour. Those found in the sinonasal tract were initially referred to as 'haemangiopericytoma-like tumours' because of their resemblance to the soft tissue haemangiopericytoma, but with several divergent pathological and clinical features (Compagno and Hyams, 1976). The origin of these rare sinonasal tumours is still debated. Recently, similarities with glomus tumour have led to the reclassification of these tumours as glomangiopericytomas. Our case further adds to this debate, as this commonly indolent, histologically uniform tumour demonstrated aggressive features with unusual immunohistochemistry.

'Pituitary palsy' - progressive neck pain and arm weakness following trans-sphenoidal hypophysectomy

\section{Grandidge, A Hall, N Kitchen}

From the National Hospital for Neurology and Neurosurgery, London

\section{Introduction}

Two years after trans-sphenoidal hypophysectomy, performed for pituitary adenoma, a 63-year-old gentleman developed intrascapular pain with progressive right arm weakness.

\section{Case report}

This otherwise well gentleman was diagnosed with atypical pituitary macroadenoma in 2010, and received 50.4 Gy radiotherapy following surgery. He presented with neck and intrascapular pain in September 2012, which was treated as osteoarthritis of the cervical spine. His pain worsened, and in December 2012, he developed right arm weakness. Magnetic resonance imaging (MRI) of the brain and cervical spine revealed a solitary lesion at the $\mathrm{C} 2$ level. This was excised; intra-operative macroscopic appearances were akin to pituitary adenoma.

\section{Radiological findings}

Pre- and post-gadolinium MRI of the $\mathrm{C} 2-3$ level revealed a focal, well-circumscribed, homogeneous, intradural extramedullary and enhancing mass, with T2-weighted high-signal intensity. The mass was in a right ventral parasagittal location, extending towards the exit foramina. There was posterolateral cord displacement, but no signal alteration.

\section{Histological findings}

Trans-sphenoidal hypophysectomy revealed atypical pituitary macroadenoma with follicle-stimulating hormone expression and increased $\mathrm{Ki}-67$ proliferative index (15-20 per cent).

The C2 lesion was a morphologically malignant neuroendocrine tumour, with a similar immunoprofile to the atypical pituitary adenoma. Findings were consistent with metastatic disease, and the lesion was regarded as pituitary carcinoma.

Dr Sandison commented that the high mitotic rate of the pituitary lesion pointed towards a carcinoma as opposed to an adenoma.

\section{Management}

Following complete excision, the patient made a good recovery. He underwent whole body imaging, and was subsequently transferred to the oncology department for chemotherapy.

\section{Discussion}

Extremely rare, metastatic disease is documented in patients with atypical pituitary adenoma. Dr Sandison noted that it would be advisable to seek the opinion of a neuropathologist with a special interest in pituitary neoplasms. This would allow further histological assessment, such as P53, to help identify those patients that may develop metastatic disease.

Dr Connor pointed out that because of the rarity of this condition, there is no evidence to recommend positron emission tomography scans for monitoring these lesions.

\section{Conclusion and take-home message}

An index of suspicion should be raised in patients presenting with symptoms of lesions within the neuraxis. Urgent investigations should be instigated, to identify metastatic disease.

\section{Death from orbital cellulitis}

L Woo, S Toma, C Hopkins

From the Guy's and St Thomas' NHS Foundation Trust, London

\section{Introduction}

Mucormycosis is a rare, aggressive, opportunistic fungal infection, most commonly caused by mucor or rhizopus spp. Early recognition, debridement, antifungals and control of co-morbidities are the mainstay of treatment. Survival rates have improved, but deaths still occur if the condition is not treated early.

\section{Case report}

A 78-year-old woman with poorly controlled diabetes was transferred for definitive management of right orbital cellulitis secondary to sinus pathology. At her local hospital, functional endoscopic sinus surgery and orbital decompression were performed for right proptosis, ophthalmoplegia and loss of vision, but the eye was retained. Two months later, she developed nausea, vomiting and confusion, prompting transfer. She was found to have developed nephrotoxicity associated with antifungal treatment. 


\section{Radiological findings}

Magnetic resonance imaging and computed tomography conducted post-transfer demonstrated orbital and sinus pathology. Repeat imaging raised a possibility of the persistence of invasive fungal infection around the right cavernous sinus.

\section{Histological findings}

Initial histology showed morphological features of mucormycosis. Repeat histology confirmed its lingering presence.

\section{Management}

Endoscopic examination and debridement revealed inflammatory changes. The patient subsequently underwent enucleation of her right orbit, and remained on systemic antifungals.

Post-operatively, the patient became confused and her general medical health deteriorated. Unfortunately, she suffered progressive renal failure and a fatal bowel perforation.

\section{Discussion}

This case reiterates the importance of early aggressive treatment, and raises the question of whether early aggressive surgery could have prevented further disease progression and subsequent systemic complications. Those at the meeting were in general agreement with this idea.

\section{Conclusion}

Despite newer developments in medical therapy, aggressive surgery remains a priority in the treatment of mucormycosis.

\section{The largest known nasal mass reported in the medical} literature?

A Roberton, A Carter, C Hopkins

From the Guy's and St Thomas' NHS Foundation Trust, London

\section{Introduction}

We report what appears to be the largest rhinophyma documented in the medical literature. Previous reports have described various laser techniques for excising such large rhinophymas; we describe a much simpler technique involving the use of cutting diathermy, a disposable razor and adrenaline-soaked swabs.

\section{Case report}

A 60 -year-old man presented following a 10-year history of an enlarging rhinophyma. Personal circumstances had prevented him from seeking specialist advice earlier. He presented only once the size of the mass had caused impairment to his vision.

\section{Histological findings}

Histopathology confirmed the mass was a rhinophyma. There was no concomitant malignancy, which has previously been reported to occur in up to 30 per cent of cases.

\section{Management}

A facemask was unable to be placed on the patient. Consequently, the anaesthetic team performed fibre-optic intubation whilst the patient was awake. The bulk of the mass was removed using cutting diathermy. Simple disposable razors were employed to shave the remaining tissue until follicular epithelium was encountered; this resulted in a smooth contour and also allowed depth control. Adrenaline-soaked swabs minimised bleeding. The nose was dressed with layered Fibrillar ${ }^{\mathrm{TM}}$ Surgicel ${ }^{\circledR}$ dressing and secured with Tisseel fibrin glue. The patient was asked to apply chloramphenicol ointment liberally once the dressing fell off

\section{Discussion}

The weight of the mass removed was $800 \mathrm{~g}$. The post-operative haemoglobin level was $14.3 \mathrm{~g} / \mathrm{dl}$. The patient was extremely pleased with the result.

\section{Conclusion}

This case highlights the use of simple equipment, available in the vast majority of worldwide surgical departments, which achieved minimal blood loss and a good cosmetic result.

\section{Head and neck section}

Chairman: Mr Jean Pierre Jeannon

\section{Mucoepidermoid carcinoma in a thyroglossal duct remnant}

E Warner, E Odell, J P Jeannon

From the Guy's and St Thomas' NHS Foundation Trust, London

\section{Introduction}

Thyroglossal duct cysts are common midline neck swellings resulting from remnants of the thyroglossal duct. They often contain ectopic thyroid tissue. Malignant transformation has been reported, usually papillary thyroid carcinoma. Mucoepidermoid tumours typically occur in the salivary glands and only rarely in the thyroid. This is the first known case of a mucoepidermoid carcinoma occurring within a thyroglossal duct cyst or remnant.

\section{Case report}

A 73-year-old woman of Bangladeshi origin presented with an enlarging midline neck lump of 2 years duration. There were no worrying features; thyroglossal duct cyst was suspected and confirmed by cytology. Excision was recommended, but the patient declined surgery. The mass rapidly increased in size. Twenty-four months later, repeat fine needle aspiration cytology findings were highly suspicious for malignancy. A computed tomography scan showed a $5 \mathrm{~cm} \times 7 \mathrm{~cm} \times 6.6 \mathrm{~cm}$ anterior midline neck mass from the thyroid gland to the hyoid bone, which was hyperintense on T2-weighted magnetic resonance imaging. Total thyroidectomy and selective neck dissection were performed. The tumour extended to the trachea, and adjuvant radiotherapy was recommended for incomplete surgical margins.

\section{Histology}

Professor Edward O'Dell (Department of Pathology, Guy's Hospital) reviewed the histology and noted a uniform, well-circumscribed tumour, with clusters of clear and epithelial cells. There was pleomorphism, cellular atypia and mucin secretion. Professor O'Dell's diagnosis was high-grade mucoepidermoid carcinoma.

\section{Discussion}

There is no ectopic salivary tissue in a thyroglossal tract and no connection to a site with mucous glands. This appears to be a case of primary thyroid mucoepidermoid carcinoma (as recognised to rarely occur in the thyroid), found in a thyroglossal duct remnant. 
Take-home message

Thyroglossal duct cysts have the potential for malignant transformation. They should be surgically excised if possible, regardless of a benign presentation.

\author{
A non-responding sore throat and swelling; when is a \\ hoof print not a horse? \\ T Kanzara, J Virk, H Kaddour \\ From the Queen's Hospital Romford, Essex
}

\section{Introduction}

Parapharyngeal space neoplasms are unusual; a particularly rare variant is synovial sarcoma. The anatomical location of these tumours lends itself to a myriad of differential diagnoses, making it difficult to distinguish synovial sarcomas clinically and radiologically from more common lesions.

\section{Case report}

A 17-year-old male, with a background of asthma and family history of lymphoma, presented with a 2-month history of globus sensation, dysphonia and snoring. He was systemically well. Examination showed medialisation of the left tonsil by a large smooth swelling.

\section{Imaging}

Magnetic resonance imaging (MRI) demonstrated a large encapsulated mass, arising lateral to the left tonsil and displacing it medially. Bilateral multilevel II and III lymph nodes, and adenoidal hypertrophy, were noted. A lymphoma was suspected.

\section{Histology}

Dr Sandison showed how the lesion had effaced the tonsillar tissue, and highlighted the epithelioid cells arranged in the form of branching anastomosing trabeculae surrounding islands of spindle sarcomatoid cells, with hyperchromatic nuclei. This is suggestive of a biphasic synovial sarcoma. Chromosomal testing for SYT-SSX1 translocation confirmed the diagnosis.

\section{Management}

Transoral excision biopsy of the left parapharyngeal mass and bilateral tonsillectomy were performed. The multidisciplinary team recommended referral to a sarcoma centre, where a staging computed tomography, repeat MRI and neck ultrasound with fine needle aspiration were performed. These confirmed locoregional control of the tumour. Adjuvant doxorubicin and ifosfamide chemotherapy (six cycles), and radiotherapy (60 Gy in 30 fractions), were administered. The patient remained disease-free at 13 months.

\section{Conclusion}

Although rare, synovial sarcomas merit consideration when a patient presents with unusual masses in the head and neck. Wide local excision is crucial to their management.

\section{A very rare cause of longstanding dysphagia}

A D Dragan, H Jones, P Clarke

From the Charing Cross Hospital, London

\section{Introduction}

Rhabdomyomas are very rare benign tumours of skeletal muscle origin, more commonly of cardiac and only rarely of extracardiac origin. There have only been 30 cases of parapharyngeal rhabdomyomas described to date.

\section{Case report}

A 56-year-old gentleman presented with a 1-year history of mild dysphagia. Flexible endoscopy revealed a right parapharyngeal bulge.

\section{Radiological findings}

Magnetic resonance imaging of the neck revealed a $9.4 \mathrm{~cm}$, homogeneously enhancing, soft tissue mass along the right side of the oropharynx, extending from $\mathrm{C} 2$ to $\mathrm{C} 7$. Computed tomography scans of the chest and abdomen did not reveal any other abnormalities.

\section{Histological findings}

Histology showed lobules of closely packed, uniform polygonal cells, with eosinophilic granular cytoplasm and no nuclear atypia. There were positive findings for desmin and myoglobin, which led to the diagnosis of adult extracardiac rhabdomyoma.

\section{Management}

The patient underwent a debulking excision of the mass via a transoral approach. He made a good recovery and was discharged home. Five months later, he remained asymptomatic and on a normal diet.

\section{Discussions}

Dr Sandison emphasised the importance of careful histological evaluation, as other tumours of the head and neck may have similar histological features. Dr Siddiqui used the opportunity to remind the meeting of the association of cardiac rhabdomyoma with tuberous sclerosis, and mentioned the typical radiological features of this.

\section{Conclusion}

Parapharyngeal rhabdomyomas are extremely rare, but they should be considered in the differential diagnosis of masses in the head and neck region.

\section{A rare tumour of the submandibular salivary gland}

R Roplekar, B Fu, J Weighill

From the Royal Sussex County Hospital, Brighton

\section{Introduction}

Granular cell tumour is an unusual soft tissue tumour with the potential to arise from any organ; the submandibular gland is a rarely documented site.

\section{Case report}

A 37-year-old woman presented with a small, right, level 2 neck swelling, with no associated symptoms. After an ultrasound scan and core biopsy were performed, submandibular gland excision was recommended by the head and neck multidisciplinary team, for diagnostic and therapeutic purposes.

\section{Radiology}

The ultrasound scan showed a round superficial mass between the tail of the parotid gland and submandibular gland. The suggested differential diagnoses included a tumour originating from the parotid tail (e.g. pleomorphic adenoma), the submandibular gland, accessory salivary gland or a lymph node. 


\section{Histopathology findings}

A core biopsy demonstrated lesional cells containing digested periodic acid-Schiff positive granules, which showed positivity for S100 on immunohistochemistry. There was no overt cytological atypia, rather the typical morphological features of a granular cell tumour or Abrikossoff's tumour. Dr Sandison noted that granular cell tumours, when located in the tongue, tend to be multifocal, and observation alone is an acceptable management option. In the event of malignant transformation, however, rapid growth with central necrosis is frequently observed.

\section{Management}

The excised lesion was consistent with the biopsy findings, but excision was incomplete.

At the three-week follow up, the patient was well. She continues with regular follow up in view of the incomplete excision margins, because of the likelihood of recurrence and the rare possibility of malignant transformation.

\section{Discussion and conclusion}

Dr Siddiqui advised that magnetic resonance imaging, perhaps performed pre-operatively, would have provided a baseline with which to compare future scans. Dr Sandison highlighted the merits of pre-operative, image-guided biopsies in diagnostic and treatment decision making.

Mr Jeannon agreed that regular surveillance is the correct and prudent strategy for granular cell tumour, and reminded the meeting that wide local excision of the submandibular gland should always be performed if a tumour of the gland is suspected. This reduces the likelihood of residual tumour if histology subsequently reveals malignancy. Miss Chevretton asked if Mr Jeannon would also advocate surgical clearance of level $1 \mathrm{~b}$. Mr Jeannon responded that he would only do so for this patient if their symptoms recurred.

Thymic teratoma masquerading as a thyroid lump

W S Cho, R S Sahota, P J Conboy

From Leicester Royal Infirmary

\section{Introduction}

Teratomas are germ cell tumours very rarely found in the neck. We present a thymic teratoma presenting as what appeared to be an intrathyroid lesion, which, to our knowledge, has not previously been described.

\section{Case report}

A 24-year-old woman presented with an asymptomatic neck lump in the thyroid region. She underwent three ultrasoundguided aspirations that were inconclusive (Thy 1). We proceeded to perform a diagnostic right hemithyroidectomy. Intra-operatively, a multi-cystic mass containing hair and fluid was found, which extended retrosternally, and was adherent to the thyroid and right carotid artery. Incisional biopsy was undertaken and further imaging was requested. An uneventful full excision was subsequently performed by thoracic surgeons.

\section{Radiological findings}

A computed tomography (CT) scan conducted after the first operation showed an infiltrative, ill-defined, soft tissue abnormality in the superior mediastinum, extending into the lower aspect of the thyroid. Dr Siddiqui commented that the radiological presence of fat within the lesion indicates either a lipoma or a teratoma. The fact that the lesion was in the thoracic cavity makes teratoma the likely diagnosis.

\section{Histological findings}

The excised specimen was a benign mature thymic teratoma, with cystic spaces lined by keratinised squamous epithelium containing hair shafts, with some benign thymic tissue.

\section{Discussion}

Dr Siddiqui and Mr Jeannon suggested that if retrosternal extension or adherence to the carotid artery had been identified from ultrasound scanning and the patient had undergone a CT scan, a diagnosis of teratoma may have been possible pre-operatively, and a single definitive procedure could have been planned in advance.

\section{Conclusion}

Although patients undergo imaging studies to guide surgery, the surgeon must always be prepared for the unexpected. A multidisciplinary team approach is the best option for difficult congenital cases.

\section{Paediatric section}

Chairman: Mr Ian Hore

\section{Recurrent epistaxis in a nine-year-old boy - benign or} malignant?

\section{S Hadjisymeou, H Dean, I Hore}

From the Evelina Children's Hospital, Guy's and St Thomas' NHS Foundation Trust, London

\section{Introduction}

We report the case of an atypical, aggressive and locally advanced tumour in a nine-year-old boy.

\section{Case report}

The patient presented to a local London hospital with a sixmonth history of right-sided, intermittent epistaxis, which had persisted despite multiple treatments with Naseptin ${ }^{\circledR}$ nasal cream and silver nitrate cautery. The patient denied any nasal obstruction or other systemic symptoms. A biopsy of an incidental postnasal space mass had been performed prior to transfer.

\section{Radiology}

A magnetic resonance imaging (MRI) scan of the head and neck revealed a right-sided $3.5 \mathrm{~cm}$ nasoethmoid mass without intracranial or orbital extension. A computed tomography scan of the sinuses showed the cribriform plate and lamina papyracea to be intact. Chest and abdominal imaging showed no evidence of metastatic disease.

\section{Histology}

Biopsy confirmed a high-grade, poorly differentiated tumour of indeterminate origin. Dr Sandison mentioned that Professor Cyril Fischer (from The Royal Marsden Hospital) had also reviewed the histology and reached the same conclusion. The surgical specimen is under continuing analysis at Boston, Massachusetts to exclude nuclear protein in testis (NUT) gene mutation, to further delineate the tissue of origin.

\section{Management}

The patient was scheduled for a combined craniofacial resection at a specialist centre, but presented pre-surgery to the 
accident and emergency department with nasal obstruction and difficulty breathing. On review, the tumour had increased significantly in size and was protruding from the nostril. Prior to further multidisciplinary team discussion, the patient experienced a major haemorrhage and expedited surgery was performed to debulk the tumour. A post-operative MRI scan demonstrated significant progression around the skull base with dural enhancement. The patient is currently on the second cycle of cisplatin and 5-fluorouracil adjuvant chemotherapy. Following chemotherapy, regression will be reassessed prior to consideration of adjuvant radiotherapy.

\section{A rare cause of childhood stridor \\ J Barr, H Dean, G Morrison \\ From the Guy's and St Thomas' NHS Foundation Trust, London}

\section{Introduction}

Achalasia is a rare cause of stridor. Only one other case has been described in children.

\section{Case report}

A 12-year-old girl presented to the ENT clinic with an 18month history of dysphagia and noisy breathing on eating. She had no problems with aspiration or dysphonia.

Flexible laryngoscopy showed a slightly retroverted epiglottis and normal vocal fold motion. Fibre-optic endoscopic evaluation of swallowing showed normal initial swallowing, but after a number of swallows biphasic stridor occurred.

\section{Radiology}

Barium swallow findings were suggestive of achalasia, with delayed passage of contrast into the stomach.

\section{Management}

Microlaryngoscopy, bronchoscopy and upper oesophagoscopy were performed. The subglottis was normal. There was no tracheoesophageal fistula or laryngeal cleft. Rigid oesophagoscopy showed smooth, non-inflamed mucosa with a dilated oesophagus. Bronchoscopy showed 75 per cent distal tracheomalacia due to flattened cartilage rings and secondary to posterior compression associated with the redundancy of oesophageal mucosa.

Oesophageal manometry showed incomplete relaxation of the lower oesophageal sphincter and absent peristalsis, which are diagnostic features of achalasia. Our patient was referred to the paediatric surgeons for consideration of Heller's myotomy. This procedure was performed and her symptoms are much improved.

\section{Discussion}

Degeneration of previously healthy tracheal cartilage can occur as a result of extrinsic compression from the oesophagus, which leads to tracheomalacia. When our patient ate, her oesophagus filled with food, causing compression of her trachea, which resulted in stridor.

\section{Conclusions}

Stridor caused by achalasia is an extremely rare finding in children; however, it is important to be aware of this condition as it is amenable to surgical treatment.
Congenital hairy polyp of the oropharynx causing airway compromise

C Vaughan, R Sharma, J McPartland

From Alder Hey Children's Hospital, Liverpool

\section{Introduction}

Hairy polyps are the commonest congenital lesions of the nasopharynx. They are benign, but can result in feeding difficulties and airway distress.

\section{Case report}

A term male neonate suffered from a cyanotic episode shortly after birth. However, on examination, no abnormality was detected at this time. Subsequently, the baby vomited, and a mass was seen protruding from the mouth. The lesion was pedunculated, and attached to the upper pole of the left tonsil. The baby was intubated and transferred to the tertiary paediatric centre.

\section{Radiology}

A magnetic resonance imaging scan with contrast was performed. An ellipsoid mass was noted filling the nasopharynx, with no communication to the skull base. The high-signal suppression on short tau inversion recovery sequences suggested the lesion was fatty.

\section{Histology}

Macroscopically, the distal segment of the lesion was discoloured. Microscopically, the surface was covered by stratified squamous epithelium, with skin adnexal structures and a core of adipose tissue. Approximately half the sample showed signs of infarction. At the junction between the infarcted and non-infarcted tissue, the surface epithelium was ulcerated, suggesting torsion.

\section{Management}

The lesion was excised whole. No recurrence was observed over the following eight months.

\section{Discussion}

The aetiology of hairy polyps remains unclear. Dr Sandison considered hairy polyps to be a form of primitive teratoma, although some pathologists are of the opinion that they may represent branchial arch malformations.

\section{Conclusion}

Hairy polyps are benign congenital tumours, but they have the potential to cause airway obstruction and death. Care must be taken in a cyanotic neonate to examine the airway thoroughly and urgently.

\section{Airway obstruction due to infectious mononucleosis recurrence}

\section{R Mehdi, M S Atfeh, H S Khalil}

From the Plymouth Hospitals NHS Trust, Plymouth University Peninsula Schools of Medicine and Dentistry

\section{Introduction}

Infectious mononucleosis syndrome is a multi-systemic manifestation of the viral infection caused by the Epstein-Barr virus. Recurrence or reactivation of the infection is uncommon and usually asymptomatic. Tonsillectomy has been considered as a means to prevent reactivation of the disease. 


\section{Case history}

A 15-year-old boy presented with a 7-day history of sore throat and dysphagia, with a previous history of tonsillectomy for recurrent tonsillitis. The patient also experienced purulent rhinorrhoea, intermittent epistaxis, neck stiffness, malaise and photophobia. Following discussion with the paediatric team, computed tomography (CT) scans were obtained to rule out meningitis, and a lymph node biopsy was considered to rule out lymphoma, but the latter was not performed. The patient had a positive monospot test result and elevated alanine aminotransferase levels (115 IU/1). Fibre-optic nasendoscopy revealed hypertrophic lymphoid tissue in the postnasal space. The patient was subsequently managed for recurrent infectious mononucleosis with steroids, steroids and symptomatic relief.

\section{Radiology}

The CT scan showed hypertrophic lymphoid tissue in the postnasal space causing complete occlusion of the choana and abutting of the soft palate.

\section{Conclusion}

This case highlights the possibility of recurrent infectious mononucleosis following tonsillectomy. The decision to not perform lymph node biopsy was supported during the meeting.

A rare multi-system genetic disease causing functional and cosmetic nasal deformity

D Patel, P Modayil, G A Morrison

From the Evelina Children's Hospital, Guy's and St Thomas' NHS Foundation Trust, London

\section{Introduction}

Tuberous sclerosis complex is a genetic disorder affecting cellular differentiation, proliferation and migration early in development, resulting in a variety of hamartomatous lesions that may affect virtually every organ system of the body.

\section{Case report}

A 15-year-old boy with tuberous sclerosis, which affected the skin of his face and nose, presented with a lesion involving the left alar region, causing a tumourous external nasal deformity. The lesion also involved the vestibular skin, causing severe left-sided nasal obstruction. The above conditions had a major impact on the patient's quality of life. Before referral to the Evelina Children's Hospital, the patient had received laser treatment to debulk the mass.

The patient underwent surgery via a nasal rim incision, to debulk the tumour both internally and subcutaneously over the alar region. The lower lateral alar cartilage had been completely destructed. Rhinoplasty reconstruction was undertaken using sculptured septal cartilage. Post-operative results showed good healing with alar support and contour, and a good nasal airway.

Histology

Histology was reported as showing no diagnostic features.

Radiology

Dr Siddiqui noted evidence of cortical tubers on the magnetic resonance imaging scan of the patient's brain. He also reminded the meeting of the strong association between angiomyolipomas and tuberous sclerosis.

\section{Discussion}

Dr Sandison advised that the diagnosis of nasal tuberous sclerosis lesion requires the identification of certain clinical and radiological features to support histological diagnosis.

\section{Conclusion and take-home message}

Surgical clearance of tuberous sclerosis lesions is sometimes required to achieve a good functional and cosmetic outcome. 